\title{
Bulk motion and collimation of Gamma Ray Bursts
}

\author{
Giancarlo Ghirlanda* \\ INAF - Osservatorio Astronomico di Brera, Via E. Bianchi 46, I-23807 Merate (LC) Italy \\ E-mail: giancarlo.ghirlanda@brera.inaf.it
}

\begin{abstract}
Gamma Ray Bursts are the most luminous extragalactic sources in the Universe. Theoretical arguments and direct observational evidences suggest that they are powered by relativistic jets with typical aperture of few degrees. The bulk Lorentz factor of the material outflowing through these jets can attain extreme values (up to 1000). Despite the direct measure of these two parameters $\left(\theta_{\mathrm{j}}\right.$ and $\left.\Gamma_{0}\right)$ is difficult, their characteristic values and the possible link between them are fundamental to understand the physical processes of GRB and their origin. The current status and debate on our knowledge of the collimation and bulk motion in GRB jets is discussed.
\end{abstract}

Frontier Research in Astrophysics,

26-31 May 2014

Mondello (Palermo), Italy

*Speaker. 


\section{Introduction}

Gamma Ray Bursts (GRB - see Kumar \& Zhang 2014 for a recent review) are the most luminous extragalactic sources detected up to extreme distances $(z=8.2$, Salvaterra et al. 2009; Tanvir et al. $2009-z \sim 9.2$, Cucchiara et al. 2011). Long GRBs (with observer-frame duration $\geq 2 \mathrm{sec}$ ) are thought to originate from the core collapse of massive stars while the origin of short bursts is identified with the coalescence of two compact objects. In both cases, accretion onto the newly-born compact object (very likely a $\mathrm{BH}$ ) powers a relativistic outflow in the form of two opposite jets. GRBs are jetted and relativistic sources: their emission (from the $\gamma$-rays to the radio wavelengths) originates within a cone of aperture $\theta_{\mathrm{j}} \sim 1^{\circ}-5^{\circ}$ and it is produced by dissipation (e.g. relativistic shocks and/or magnetic reconnection) within an outflow expanding with typical bulk Lorentz factor $\Gamma \gg 100$.

According to the standard model of GRBs, the outflow initially undergoes a pressure-driven expansion which converts internal energy into kinetic $E_{\text {kin }}$ (acceleration phase). The prompt high energy $\gamma$-ray photons are thought to be produced by dissipation events (internal shocks - Rees \& Meszaros 1992) after the acceleration, i.e. during the coasting phase when $\Gamma=\Gamma_{0}=$ const. The outflow expands in the interstellar medium, increasing its mass and decelerating ( $\Gamma$ decreases with time). The afterglow emission is produced by the dissipation of the kinetic energy which is left after the prompt phase (Meszaros \& Rees 1997).

The radiation we see from GRBs is amplified by relativistic beaming. During the prompt phase, the beaming angle is smaller than the jet opening angle, i.e. $1 / \Gamma_{0} \ll \theta_{\mathrm{j}}$, for typical values of these two parameters. The observer cannot perceive the presence of a jet from the observation of the prompt $\gamma$-ray emission (Rohads 1997). The signature of the jet appears in the afterglow emission when, due to the deceleration of the blast wave, $1 / \Gamma_{0} \sim \theta_{\mathrm{j}}$. The measure of this characteristic time $t_{\mathrm{j}}$ (the jet break) allows us to infer the opening angle of the jet $\theta_{\mathrm{j}}$. The transition between the coasting and the deceleration phase should produce a peak in the afterglow light curve: the afterglow luminosity increases proportional to the emitting surface during the coasting phase until the decrease of $\Gamma$, due to the deceleration, overwhelms it. The measure of this characteristic peak time $t_{\mathrm{p}}$ (the afterglow onset) allows us to estimate the bulk Lorentz factor $\Gamma_{0}$ (Sari \& Piran 1999) before the start of the deceleration, i.e. during the coasting phase, which corresponds to the maximum (average) value the outflow attained during its dynamical evolution.

\section{GRB jets}

The presence of jets in GRBs is expected for the nature of the central engine (i.e. most likely a spinning $\mathrm{BH}$ ) and it is "invoked" to reduce the huge isotropic equivalent energy derived from the prompt emission observations (up to $E_{\text {iso }} \sim 5 \times 10^{54} \mathrm{erg}$ of isotropic equivalent energy). The isotropic equivalent energy $E_{\text {iso }}$ (or luminosity $L_{\text {iso }}$ ) of $\sim 200 \mathrm{GRBs}$ with measured $z$ is broadly dispersed over more than three orders of magnitudes. This spread is reduced correcting the isotropic quantities for the collimation factor, i.e. $E_{\gamma}=E_{\text {iso }}\left(1-\cos \theta_{\mathrm{j}}\right)$ and $L_{\gamma}=L_{\text {iso }}\left(1-\cos \theta_{\mathrm{j}}\right)$. Frail et al. (2001) found a clustering of $E_{\gamma} \sim 5 \times 10^{50} \mathrm{erg}$ based on a small sample of 17 events. This clustering, however, was not enough to use GRBs as standard candles (Bloom et al. 2003). The discovery of a tight (less than $0.07 \mathrm{dex}$ ) correlation between the collimation corrected energy $E_{\gamma}$ 
and the rest frame peak energy $E_{\text {peak }}$ of the prompt emission $v F_{v}$ spectrum (Ghirlanda, Ghisellini \& Lazzati 2004; 2007) allowed us to use, for the first time, GRBs as a new cosmological tool (Ghirlanda et al. 2004a).

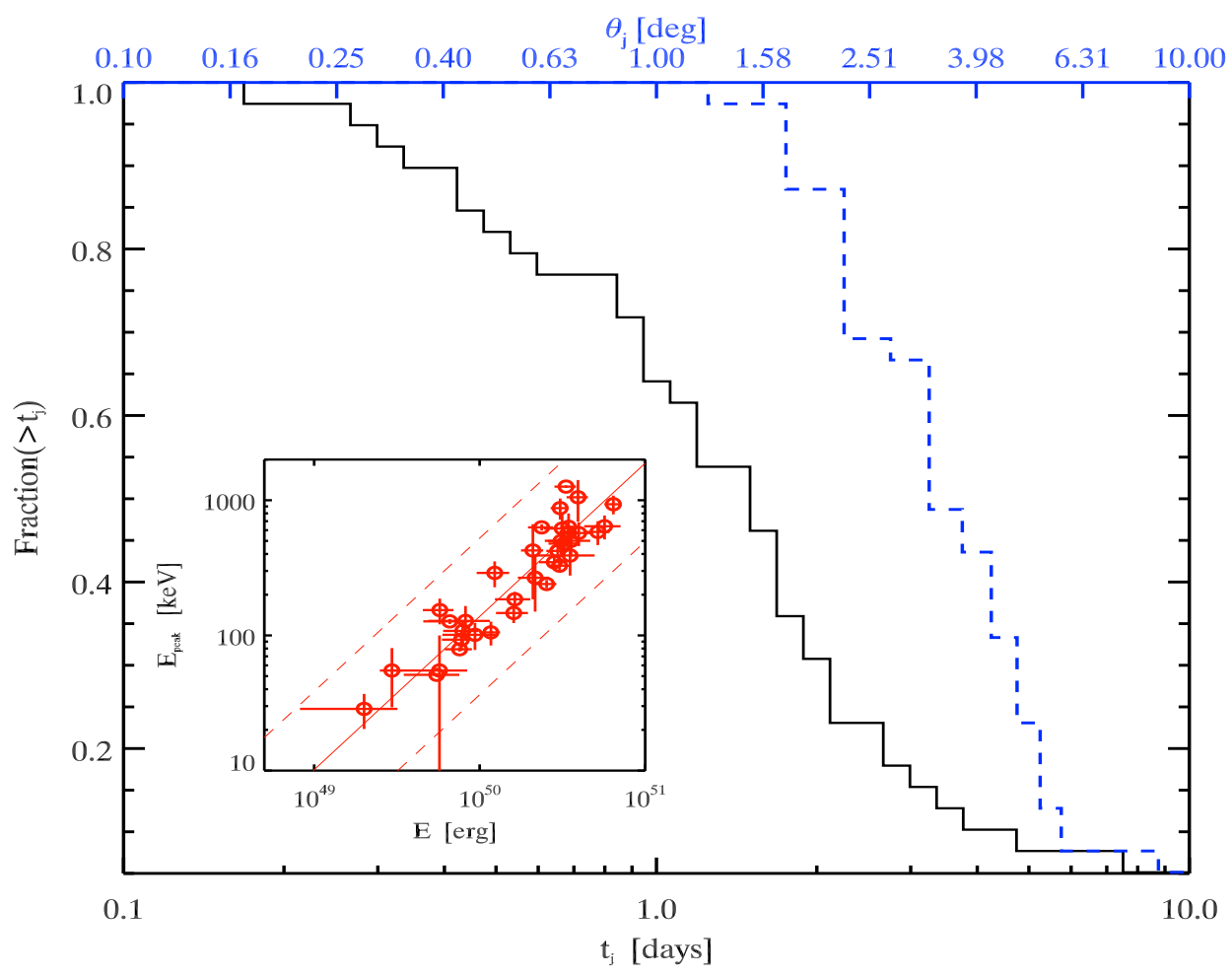

Figure 1: Cumulative distribution (solid black line) of jet break time $t_{\mathrm{j}}$ (as measured in the observer frame). The dashed blue line shows the distribution of the jet opening angles (derived under the assumption of a wind density profile). The corresponding values are shown in the upper $\mathrm{x}$-axis. In the inset it is shown the correlation between the collimation corrected energy of GRBs (i.e. the true energetic) and the prompt emission peak energy. The $3 \sigma$ dispersion around the correlation is shown by the dashed red lines.

The measure of $t_{\mathrm{j}}$ from the optical afterglow light curves shows that this is typically between 0.3 and 10 days after the prompt emission. From $t_{\mathrm{j}}$ we can estimate the jet opening angle $\theta_{\mathrm{j}} \propto$ $\left(A t_{\mathrm{j}} / E_{\mathrm{k}, \text { iso }}\right)^{1 / 4}$. Here we consider a wind density profile, scaling as $R^{-2}$ with the distance $R$ from the central engine, with normalization $A=3.15 \times 10^{35} \mathrm{~cm}^{-2}$ (Nava et al. 2006). Alternatively, a uniform medium has been considered (Ghirlanda et al. 2004). Under the hypothesis that $\sim 20 \%$ of the total kinetic energy is dissipated into radiation during the prompt phase (i.e. radiative efficiency $\eta=0.2), E_{\mathrm{k}, \text { iso }} \sim E_{\mathrm{iso}} / \eta$ is the kinetic energy remaining after the prompt emission phase.

A sample of 39 GRBs (updated to May 2011) with $t_{\mathrm{j}}$ measured from their optical afterglow is shown in Fig. 1 (solid line). The bursts shown here are all long: only in few short bursts the jet break has been measured. For this sample of bursts the jet break time is distributed between 0.2 days and 10 days. The relation between $\theta_{\mathrm{j}}$ and $t_{\mathrm{j}}$ shows that the larger the opening angle of the bursts, the later will be the appearance of the jet break time (for a fixed value of $E_{\mathrm{k}, \text { iso }}$ ). The average value of the distribution is $\left\langle t_{\mathrm{j}}\right\rangle \sim 1.2$ days. The corresponding jet angle distribution $\theta_{\mathrm{j}}$ is 
shown by the dashed blue line (the corresponding values are shown by the upper $\mathrm{x}$-axis of Fig. 1). The current measured $\theta_{\mathrm{j}}$ are distributed between $1^{\circ}$ and $10^{\circ}$ with an average value $\left\langle\theta_{\mathrm{j}}\right\rangle \sim 3^{\circ}$.

In the inset of Fig. 1 it is shown the updated correlation between the collimation corrected energy $E_{\gamma}$ and the peak energy of the $v F_{v}$ spectrum (open symbols). This correlation, computed under the assumption of a wind density circumburst medium, is linear and has a scatter of 0.1 dex. Further analysis and update of this sample of GRBs with measured $\theta_{\mathrm{j}}$ is presented in Ghirlanda et al. 2015 (in preparation).

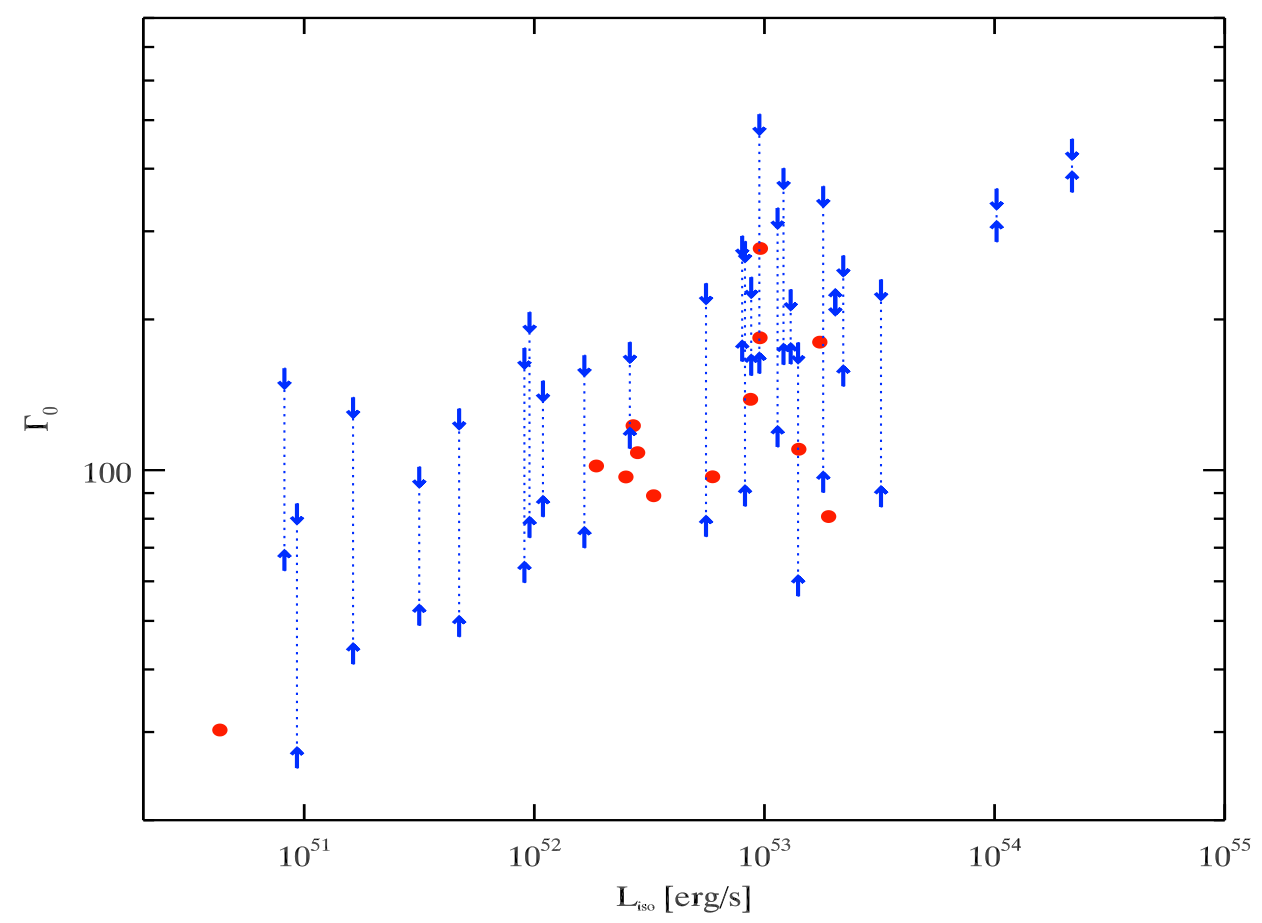

Figure 2: Bulk Lorentz factor $\Gamma_{0}$ computed under the assumption of a wind density profile for the GRBs of the Swift complete sample with an estimate of the afterglow onset time (filled red circles). Lower limits on $\Gamma_{0}$ come from upper limits on $t_{\mathrm{p}}$, upper limits on $\Gamma_{0}$ are derived assuming that $t_{\mathrm{p}} \geq t_{p, \gamma}$ where $t_{p, \gamma}$ is the peak of the prompt emission. This assumption corresponds to transfer most of the kinetic energy of the outflow (i.e. what is left after the main emission episode in the $\gamma$-ray) to the blast wave to have efficient deceleration.

\section{GRB bulk Lorentz factors}

The relativistic nature of GRBs was first suggested by the "compactness argument" (e.g. Litwick \& Sari 2001): the radiation we see should escape a compact region (as implied by the fast variability) densely populated by high energy photons (as implied by the large energetics). If not moving relativistically with bulk Lorentz factor $\Gamma \gg 100$ (Prian 1999), the pair production would suppress the spectrum at high energies (above the pair production energy threshold) contrary to the observation of high energy photons in GRBs (up to $\mathrm{MeV}$ and $\mathrm{GeV}$ energies - e.g. Ackerman et al. 2013). Direct evidence that the outflow in GRB jets expands at relativistic speeds came with 
the detection of radio variability in few cases (Frail et al. 1997). The ceasing of the variability a few days after the explosion, if interpreted as due to scintillation by interstellar clouds, allows us to infer the expansion velocity which turned out to be relativistic.

The afterglow luminosity increases with the distance from the central engine (due to the increase of the emitting surface) during the coasting phase. When the deceleration starts, however, $\Gamma$ decreases with the radius and overcomes the increase of the emitting surface. Therefore, the afterglow light curve should present a peak, i.e. the afterglow onset, $t_{\mathrm{p}}$. Early follow up of the afterglow emission can secure the measurement of this peak (e.g. Molinari et al. 2007). Through the standard model, the bulk Lorentz factor $\Gamma_{0} \propto\left(E_{\mathrm{k}, \text { iso }} / A t_{\mathrm{p}}\right)^{1 / 4}$. This is $\Gamma_{0}$ i.e. that at the end of the coasting phase which corresponds to the beginning of the deceleration. This represents the maximum $\Gamma$ that the burst attained during its dynamical evolution, though it should be regarded as an average value: it is still possible that different parts of the outflow travel with larger/smaller velocities during the coasting phase (indeed this is a requirement for the development of internal shocks). The parameter $A$ is the normalization of the density profile (assumed to scale as a wind like in the case of $\theta_{\mathrm{j}}$ above).

Since everything we observe in GRBs is subject to relativistic beaming effects, i.e. the luminosities and frequencies are boosted by $\Gamma^{2}$ and $\Gamma$ respectively, the knowledge of this parameter allows us to derive the comoving frame properties of these sources.

Ghirlanda et al. (2012) considered a sample of 30 long GRBs with a peak in their optical afterglow light curves and derived $\Gamma_{0}$ under the hypothesis of a wind density medium (the same assumption made above) or considering the homogeneous density case. Among the several results reported in that paper, they found (a) a tight correlation between the isotropic luminosity $L_{\text {iso }}$ and $\Gamma_{0}\left(L_{\text {iso }} \propto \Gamma_{0}^{2}\right)$ and (b) a clustering (mostly evident when considering the wind density profile and the luminosity) of the comoving frame luminosities around $\sim 1.5 \times 10^{48} \mathrm{erg} / \mathrm{s}$. These results led to propose a dynamical interpretation of the $E_{\text {peak }}-L_{\text {iso }}$ correlation as due to a sequence of $\Gamma_{0}$ in alternative to existing ones (Yamazaki, Ioka \& Nakamura 2004; Lamb, Donaghy \& Graziani 2005; Levinson \& Eichler 2005; Rees \& Meszaros 2005; Toma, Yamazaki \& Nakamura 2005; Thompson 2006; Giannios \& Spruit 2007; Thompson, Meszaros \& Rees 2007).

\section{Discussion and Conclusions}

The measure of $t_{\mathrm{j}}$ requires the follow up of the GRB optical afterglow until late times: $t_{\mathrm{j}}$ is typically a smooth transition (van Eerten et al. 2011) which is better observed in the light curves if early and late times observations are available. The measure of $t_{\mathrm{j}}$ is complicated by the presence in the optical light curves of several other breaks: early breaks, e.g. before 0.2 days, could be erroneously interpreted as jet breaks biasing the $\theta_{\mathrm{j}}$ distribution towards extremely small values. Indeed, in several X-ray light curves and less often in the optical, the observed emission has a flat phase (observed between 100 and $10^{5} \mathrm{~s}$ ) which is most likely not due to the afterglow and its late time break should not be used to infer $\theta_{\mathrm{j}}$ (Nava et al. 2006). It should also be noted that several authors have been using the $\mathrm{X}$-ray light curve to measure $t_{\mathrm{j}}$. This should be done with care: the $\mathrm{X}-$ ray emission (especially in the early stage) is most likely dominated by a component which is not the afterglow but rather some form of internal dissipation (e.g. Ghisellini et al. 2009). In this view, secure estimate of $t_{\mathrm{j}}$ are those based on the optical light curve, which instead has more often the 
characteristic signatures of the afterglow component. However, the distribution of $t_{\mathrm{j}}$ might not be strongly missing low values (i.e. small $\theta_{\mathrm{j}}$ ) because the recent years have witnessed an extraordinary increase of robotic telescopes which often can follow up the afterglow emission in the optical band starting relatively early after the GRB trigger. If a large fraction of the afterglows would have a jet break before 0.3 hours we should have seen it through these observations. This argument suggests that the small end of the distribution of $\theta_{\mathrm{j}}$ is relatively well constrained. On the other extreme, the measure of large $t_{\mathrm{j}}$ is complicated by the faintness of the afterglow emission at late times and by the possible contribution of the host galaxy and SN component. Therefore the large values end of the $t_{\mathrm{j}}$ distribution (i.e. corresponding to large $\theta_{\mathrm{j}}$ ) might be still only partly explored. The answer to this question can be achieved through population studies of GRBs (Ghirlanda et al. 2013) or through direct modeling of the $t_{\mathrm{j}}$ distribution accounting for the above mentioned selection biases (Ghirlanda et al. 2015).

The possible selection biases acting on the distribution of $\theta_{\mathrm{j}}$ also affect the $E_{\text {peak }}-E_{\gamma}$ correlation: the possible lack of relatively large $\theta_{\mathrm{j}}$ would impact on the right hand side of the correlation which might have a larger scatter than measured. Another issue could be related to the parent correlation $E_{\text {peak }}-E_{\text {iso }}$ (involving the isotropic equivalent energy) from which the $E_{\text {peak }}-E_{\gamma}$ correlation is derived. It has been proposed that the $E_{\text {peak }}-E_{\text {iso }}$ correlation is missing dim GRBs because they lie below the detection threshold of current GRB detectors. These events should populate the low $E_{\text {iso }}$ - intermediate $E_{\text {peak }}$ space (Band \& Preece 2005; Nakar \& Piran 2005; Shahmoradi $\&$ Nemiroff 2011; Collazzi et al. 2012; Kocevski 2012). Moreover, a possible selection bias related to the measure of $z$ acting on the $E_{\text {peak }}-E_{\text {iso }}$ correlation has been claimed (Heussaff, Atteia, Zolnierowski, 2013).

Salvaterra et al. (2012) built a flux limited sample of 58 bursts detected by the Burst Alert Telescope (BAT) on board the Swift satellite (called BAT6). The sample is selected for having a relatively large peak flux (integrated in the $15-150 \mathrm{keV}$ energy range) $P \geq 2.6 \mathrm{ph} \mathrm{cm}^{-2} \mathrm{~s}^{-1}$. This sample, after selection, results 95\% complete in redshift. Therefore, the BAT6 sample is suited to study the intrinsic properties of GRBs independently from instrumental selection biases (due to the high-flux selection which is six times larger than the average Swift threshold) and from the measure of $z$. The BAT6 sample has been used to characterize the GRB luminosity function and redshift distribution (Salvaterra et al. 2012); to study the X-ray properties (D'Avanzo et al. 2012; Campana et al. 2012) and optical characteristics (Melandri et al. 2012; Covino et al. 2012) of GRBs. Nava et al. (2012) probe the $E_{\text {peak }}-E_{\text {iso }}$ correlation with the BAT6 sample showing that the correlation is statistically significant and has a similar slope (and slightly different normalization) with respect to that defined by the larger (incomplete and heterogeneous) sample of GRBs with $z$ detected also by other instruments (or by Swift but not included in the BAT6 selection). This result is further supported by numerical population studies based on the BAT6 sample (Ghirlanda et al. 2012a).

$t_{\mathrm{p}}$ is typically measured between few tens of seconds and $1 \mathrm{~h}$ after the trigger. An extension to the lowest values of $t_{\mathrm{p}} \leq 10 \mathrm{~s}$ is possible by including (Ghirlanda et al. 2012) GRBs detected by the Large Area Telescope (LAT) on board Fermi. These few events, indeed, have been observed early on during the prompt phase and show a high energy $(\mathrm{GeV})$ tail of emission which lasts much longer than the softer emission component detected in the $\mathrm{MeV}$ range. The most common interpretation of this high energy emission is that it is produced at the forward external shock (i.e. it is afterglow - see e.g. Nava et al. 2014). In this case the peak observed in the GeV light curve can be used 
to estimate the largest $\Gamma_{0}$ (being these Fermi bursts those with the earliest $t_{\mathrm{p}}$ measured). These events show that the distribution of $\Gamma_{0}$ can extend to very large values (up to 1000). However, the detection of such early $t_{\mathrm{p}}$ is limited, despite the efforts and relevant advances brought by the network of small robotic telescopes which can follow up the optical afterglow since the early phases (i.e. a few minutes after the trigger). This suggests that the present distribution of $\Gamma_{0}$ could miss extremely large values. On the other extreme there seems to be no strong bias against the detection of late time $t_{\mathrm{p}}$. Indeed, the robotic telescopes can follow the optical afterglow up to days after the trigger and after 0.5 days the follow up by larger facilities often comes in. In general, if the optical peak is not detected within few hours after the trigger it does not comes up later. This suggests that $t_{\mathrm{p}}$ is typically localized within a few hours (at most) since the burst trigger. It has been argued (Hascoet et al. 2014) that the current sample of GRBs with measured $\Gamma_{0}$ is biased (missing early $t_{\mathrm{p}}$ corresponding to large $\Gamma_{0}$ ) and that the $L_{\text {iso }}-\Gamma_{0}$ correlation is biased since it misses large values of $\Gamma_{0}$ at almost all luminosities.

We consider the BAT6 sample (taking advantage of its high level of completeness in redshift and unbiased selection in flux): Ghirlanda et al. (2015) finds 13 GRBs of the BAT6 with measured $t_{\mathrm{p}}$ and 25 events with an upper limit. The latter are bursts which have been followed in the optical within a few hours since the burst trigger and show a decaying optical light curve. The earliest optical observation is assumed as an upper limit on $t_{\mathrm{p}}$. In Fig. 2 the correlation between the bulk Lorentz factor $\Gamma_{0}$ and the isotropic luminosity $L_{\text {iso }}$ is shown for the 13 GRBs of the BAT6 sample with measured $t_{\mathrm{p}}$. The upper limits on $t_{\mathrm{p}}$ gives lower limits on $\Gamma_{0}$. However, in order to test the reliability of this correlation and whether the plane is uniformly filled with points above the detection (circles) and the lower limits due to our limitation in measuring early $t_{\mathrm{p}}$, we need to set also an upper limit on $\Gamma_{0}$. One possibility comes from the theory: the afterglow deceleration happens once the outflow has transferred its kinetic energy to the blast wave. This is possible only after the main emission episode, where most of the prompt emission is localized. Therefore, it is plausible to assume that the peak of the afterglow should happen after the main emission episode of the prompt. Setting $t_{\mathrm{p}} \geq t_{\mathrm{p}, \gamma}$ (where $t_{\mathrm{p}, \gamma}$ is the time of the peak of the prompt $\gamma$-ray emission) we can derive an upper limit on $\Gamma_{0}$. These are represented in Fig. 2 and are connected for the same bursts with the corresponding upper limits. Noteworthy, the upper/lower limits define intervals which follow the same correlation of the measurements. This is indicative that the correlation between $\Gamma_{0}$ and the luminosity is real and not induced by selection effects on the estimate of $t_{\mathrm{p}}$. Through a statistical analysis which considers also the upper/lower limits on $\Gamma_{0}$ we find that the correlation is significative at $>3 \sigma$ and that its slope should be $0.30 \pm 0.02$.

Acknowledgments A Prin-INAF 2011 (1.05.01.90.15) is acknowledged for financial support.

\section{References}

[1] Kumar P. \& Zhang B., 2014, Physics Reports, arXiv:1410.0679

[2] Salvaterra R., Della Valle M., Campana S., et al., 2009, Nature, 461, 1258

[3] Tanvir N., Fox D. B., Levan A. J., et al., 2009, Nature, 461, 1254

[4] Cucchiara A., Levan A. J., Fox D. B., et al., 2011, ApJ, 736, 7 
[5] Rees M. J. \& Meszaros P., 1992, MNRAS, 258, 41

[6] Meszaros P. \& Rees M. J., 1997, ApJ, 476, 232

[7] Rhoads J. E., 1997, ApJ, 487, L1

[8] Sari R. \& Piran T., 1999, ApJ, 520, 641

[9] Frail D. A., Kulkarni S. R., Sari R., et al., 2001, ApJ, 526, L55

[10] Bloom J. S., Frail D. A., Kulkarni S. R., 2003, ApJ, 594, 674

[11] Ghirlanda G., Ghisellini G., Lazzati D., 2004, ApJ, 616, 331

[12] Ghirlanda G., Nava L., Ghisellini G., et al., 2007, A\&A, 466, 127

[13] Ghirlanda G., Ghisellini G., Lazzati D. et al., 2004a, ApJ, 613, L13

[14] Nava L., Ghisellini G., Ghirlanda G., et al., 2006, A\&A, 450, 471

[15] Lithwick Y. \& Sari R., 2001, ApJ, 704, 1405

[16] Piran T., 1999, PhR, 314, 575

[17] Ackermann M., Ajello M., Asano K., et al., 2013, ApJS, 209, 11

[18] Frail D., Kulkarni S. R., Nicastro, L., et al., 1997, Nature, 389, 261

[19] Molinari E., Vergani S. D., Malesani D., et al., 2007, A\&A, 469, L13

[20] Ghirlanda G., Nava L., Ghisellini G., et al., 2012, MNRAS, 420, 483

[21] Yamazaki R., Ioka K., Nakamura T., et al., 2004, ApJ, 606, L33

[22] Lamb D. Q., Donaghy T. Q., Graziani C., 2005, ApJ, 620, 355

[23] Levinson A. \& Eichler D., 2005, ApJ, 629, L13

[24] Rees M. J. \& Meszaros P., 2005, ApJ, 628, 847

[25] Toma K., Yamazaki R., Nakamura T., 2005, ApJ, 635, 481

[26] Thompson C., 2006, ApJ, 651, 333

[27] Giannios D. \& Spruit H. C., 2007, A\&A, 496, 1

[28] Thompson C., Mesazaros P. \& Rees M. J., 2007, ApJ, 666, 1012

[29] van Eerten H. J., Meliani Z., Wijers R. A. M. J., et al., 2011, MNRAS, 410, 2016

[30] Ghisellini G., Nardini M., Ghirlanda G., et al., 2009, MNRAS, 393, 253

[31] Ghirlanda G., Ghisellini G., Salvaterra R., et al., 2013, MNRAS, 428, 1410

[32] Band D. \& Preece R. D., 2005, ApJ, 627, 319

[33] Nakar E. \& Piran T., 2005, MNRAS, 360, L73

[34] Shahmoradi A. \& Nemiroff R. J., 2010, MNRAS, 407, 2075

[35] Collazzi A. C., Schaefer B. E., Goldstein A., et al., 2012, ApJ, 747, 39

[36] Kocevski D., 2012, ApJ, 747, 146

[37] Heussaff V., Atteia J.-L., Zolnierowski Y., 2013, A\&A, 557, 100

[38] Salvaterra R., Campana S., Vergani S. D., et al., 2012, ApJ, 749, 68 
[39] D’Avanzo P., Salvaterra R., Sbarufatti B., et al., 2012, MNRAS, 425, 506

[40] Campana S., Salvaterra R., Melandri A., et al., 2012, MNRAS, 421, 1697

[41] Melandri A., Sbarufatti B., D’Avanzo P., et al., 2012, MNRAS, 421, 1265

[42] Covino S., Melandri A., Salvaterra R., et al., 2012, MNRAS, 432, 1231

[43] Ghirlanda G., Ghisellini G., Salvaterra R., et al., 2012a, MNRAS, 422, 2553

[44] Nava L., Vianello G., Omodei N., et al., 2014, MNRAS, 443, 3578

[45] Hascoet R., Beloborodov A., Daigne F., et al., 2014, ApJ, 782, 5 